\title{
El aprendizaje basado en proyectos como metodología vertical integradora.
}

\author{
Verdejo Gimeno, Pedro ${ }^{a}$, Abalos Ramos, Ana ${ }^{b}$, Juan Ferruses, Ignacio ${ }^{c}$ \\ ${ }^{a}$ Universidad CEU Cardenal Herrera, Valencia, España, pverdejo@uchceu.es, ${ }^{b}$ Universidad CEU \\ Cardenal Herrera, Valencia, España, ana.abalos@uchceu.es ' Universidad CEU Cardenal Herrera, \\ Valencia, España, ignacio.juan@uchceu.es
}

\begin{abstract}
Resumen
Una de las mayores problemáticas en la docencia universitaria es, que el alumno pueda experimentar y comprender la estrecha relación y dependencia entre las diferentes áreas de conocimiento en su futura actividad profesional.

En el sistema tradicional académico, cada materia realiza exámenes o ejercicios de forma independiente, sin contemplar la incidencia que tienen unas sobre las otras. Esto motiva que el alumno en escasas ocasiones durante sus estudios pueda hacerse una idea incidencia de todas las diferentes materias en su conjunto.

Para evitar esta situación, la metodología de aprendizaje basada en resolución de proyectos puede configurarse como la mejor opción para abordar esta carencia, pero al contario de lo que vine siendo habitual donde el alumno aborda un proyecto de forma más extensiva para defenderlo y obtener su titulación al final de sus estudios, se plantea el uso de esta paulatinamente desde tercer curso, incrementando la complejidad del proyecto en base al grado de conocimiento.
\end{abstract}

En este sentido, se ha puesto en marcha una nueva asignatura desde el tercer curso del grado en Fundamentos de Arquitectura, que pretende que el alumno desarrolle un proyecto de manera multidisciplinar, atendiendo a todas las necesidades del proyecto arquitectónico y sin menospreciar su valor formal.

Bajo esta intención, se genera un espacio de encuentro y trabajo entre distintos profesores y alumnos, donde el resultado es un único proyecto en respuesta a todas las necesidades planteadas desde las áreas, pero sin perder la coherencia proyectual.

Palabras clave: ABP, aprendizaje integrado, colaborativo, 


\section{Introducción}

Decía el arquitecto Ignacio Vicens en una de sus dinámicas conferencias en la Escuela de Arquitectura de Alicante, que "la arquitectura es como una tarta de mil capas, todas de diferentes espesores, pero que cuando una de ellas fallaba toda la tarta podía caer al suelo". Con este símil tan gráfico, podemos hacernos una idea de la importancia de todas las áreas de conocimiento que integran la formación del arquitecto.

Por otro lado, la formación histórica del arquitecto en los diferentes planes de estudio desde los años 60, se ha formulado como la correlación de diferentes asignaturas dentro de cada materia que raramente se ejercitan de forma conexa en la formación del estudiante, contrariamente a lo que sucede en su futura actividad profesional. Incluso en el trabajo final de carrera como última actividad académica, los alumnos interpretan el proceso proyectual como una actividad lineal, que partiendo de una idea, da como resultado una la forma para posteriormente se introduzcan los condicionantes técnicos sin ningún tipo de vinculación, debiendo ser posteriormente el su ejercicio profesional, donde deban integrar todas las partes que conforman el proyecto arquitectónico.

Por tanto, queda evidenciado la necesidad docente de una mayor implicación y transversalidad de todas la "capas" o partes que configuraran un proyecto a la hora de su desarrollo, evitando un esporádico encuentro al final del proceso del aprendizaje del alumno y de manera casi autónoma. Esta problemática es la que hace plantear la propuesta de romper con la forma tradicional en la que se ha impartido la docencia del proyecto arquitectónico, hacia la búsqueda de una coherente relación de las partes que lo componen, permitiendo al estudiante comprobar y ejercitar la estrecha relación de convivencia y dependencia que existe entre la parte formal y la técnica, llegando incluso a plantear lo tectónico como una condición necesaria que delimita las posibilidades de la forma, (Piñón, 2006).

Con implantación del nuevo grado de Fundamentos en Arquitectura, parecía una excusa perfecta para romper esta histórica rigidez a favor de la convivencia más estrecha entre las distintas áreas de conocimiento, permitiendo la integración completa de las diferentes asignaturas a la hora de abordar un proyecto arquitectónico. El objetivo es llevar al alumno a una dimensión donde comience a ser consciente de todas las implicaciones que puedan tener la resolución constructiva, estructural y de las instalaciones, en estrecha armonía con la resolución formal del proyecto. Por tanto, se trata de romper una metodología lineal de enseñanza, por otra donde las asignaturas discurran de forma paralela y dependientes. 


\section{Planteamiento}

Para hacer efectivo que el alumno pueda comprobar la transversalidad entre las distintas áreas de conocimiento que conforman estudiar Fundamentos de Arquitectura, se ha planteado crear un nuevo escenario para que este contacto no fuera esporádico ni puntual, con la intención de que al finalizar sus estudios, los estudiantes tuvieran interiorizado la dependencia de las distintas áreas en el desarrollo del proceso proyectual.

Este nuevo escenario posibilita que los alumnos sigan una estructura clara de aprendizaje siguiendo un planteamiento reconocido y organizados en base a dos ejes, donde en primer lugar correspondería a las categorías de recordar, entender y aplicar, para que posterioremente, de forma práctica sean capaces de analizar, evaluar y crear bajo la justificación de sus acciones.

Temporalmente, a corto plazo se desarrolla en un eje horizontal con un cambio significativo en la metodología de enseñanza, transformandose desde el uso de un método inductivo y una técnica principalmente magistral, hacia un método analítico y deductivo con un aprendizaje basado en la trasnversalidad de contenidos bajo el hilo conductor de un proyecto arquitectónico. Por otro lado a largo plazo, esta estrategia de integración de asignaturas evoluciona de manera vertical en los sucesivos cursos, pero incrementando gradualmente el grado de dificultad por proponerse proyectos cada vez más complejos.

El esquema de aproximación a esta nueva metodología se inicia con el curso lectivo en su primer semestre, donde las asignaturas técnicas y de proyectos se desarrollan de forma tradicional, es decir, de manera independiente y autónoma pero bajo una coordinación de sus contenidos. De forma paralela, los alumnos desarrollarían un proyecto arquitectónico en la asignatura proyectual correspondiente, bajo un programa de necesidades y un ámbito territorial específicos. El cambio se realiza a partir del segundo semestre donde las asignaturas técnicas trasnforman su formato habitual, para pasar a integrase en una nueva asignatura puramente práctica y que tiene como objetivo físico el desarrollo técnico del proyecto desarrollado por el propio alumno el semestre anterior. 

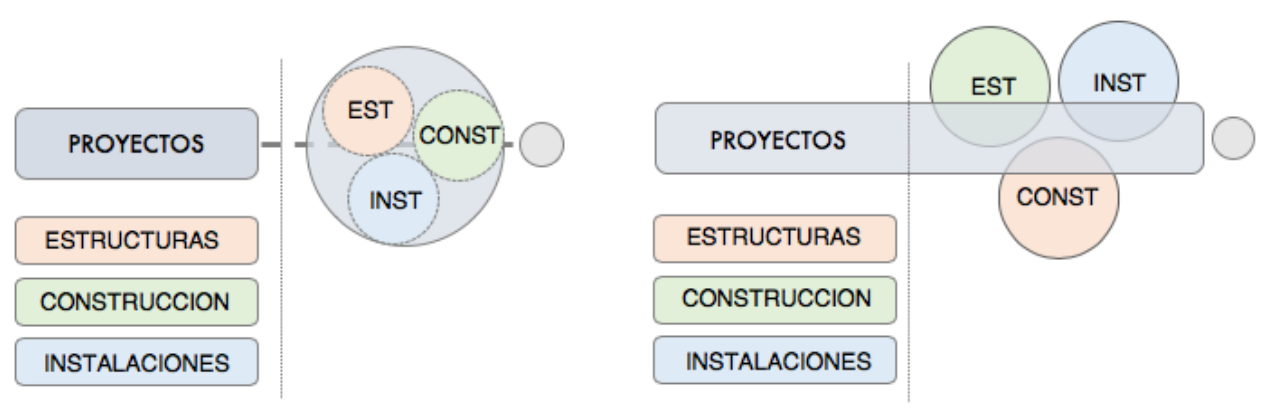

Fig. 1 Esquemas de la implantación horizontal en el primer cusro y en los sucesivos.

La intención en este primer acercamiento se basa en que el alumno comprenda y experimente la dificultad que tiene resolver técnicamente un proyecto si no se han tenido en cuenta a priori todas sus implicaciones. Basado en la técnica del aprendizaje práctico con su propio proyecto, los estudiantes comprenden los errores cometidos inicialmente mediante la evaluación de su propuesta analizandola desde un posicionamiento técnico, para realizar las modificaciones oportunas para dar respuesta a todos los requisitos. Es posible afirmar que en el primer curso donde se implanta esta metodología, los alumnos realizarían un aprendizaje que podría basarse en la decepción, debido principalmente porque empiezan a ser conscientes de que ningún proyecto es viable sin tener que variar sustancialemnte su propuesta inicial. (Azula, 1988). Por tanto podríamos situarnos en los últimos niveles de la taxonomía del Bloom en referencia a evaluar y cambiar o crear.

Para favorecer un aprendizaje significativo de la incidencia de todas las áreas de conocimiento en el resultado del proyecto, es necesario utilizar una herramienta ágil y flexible, acorde a los tiempos actuales. Por ello se prioriza el uso de un sistema BIN o sistema integrado de información, que exigen al estudiante que cualquier cambio o modificación realizado en alguno de los ámbitos del proyecto, inciden de forma inmediata sobre todo su conjunto. (Leon, 2016)

La organización vertical de la metodología, además de suponer un incremento gradual en la complejidad de los programas de las asignaturas proyectuales, contempla principalmente romper con la liealidad a la hora de resolver un proyecto arquitectónico. Este aspecto contempla na necesidad de combinar tanto las asignaturas técnicas como las proyectuales, apareciendo unnuevo espacio de proyectos integrados, donde todas las materias, técnicas y proyectuales, conviven paralelamente hacia la resolución del proyecto experimentando el verdadero cambio metodológico. 


\section{Experiencia}

Este nuevo planteamiento de entender la resolución del proyecto arquitectónico se puso en marcha en el tercer curso de grado de Fundamentos en Arquitectura, siguiendo la estructura horizontal y vertical planteada.

En el primer semestre los alumnos inician su actividad proyectual con un proyecto arquitectónico abordado expresamente desde la asignatura de proyectos. En este ejercicio, además de dar respuesta a un determinado programa de necesidades en un emplazamiento concreto, deberán tener en cuantas una serie de limitaciones en cuanto volúmenes, luces y complejidad espacial coordinadas con las asignaturas técnicas. Su intención, es favorecer un posterior desarrollo ágil, coherente y que asegure una dificultad razonable en su resolución tectónica. De forma paralela las asignaturas técnicas se siguen impartiendo independiente combinando método y forma tradicionales.

En el segundo semestre es donde se integran las asignaturas técnicas para posibilitar el desarrollo técnico del proyecto que el alumno ha desarrollado con anterioridad. La intención de utilizar un proyecto propio del alumno es que se encuentre más motivado e implicado a la hora de tener que realizar modificaciones, ya que en todo momento deben de evitar la perdida de su propuesta original.

La aparición de nuevos pilares en lugares poco deseados, la necesidad de espacios para albergar los cuartos técnicos de instalaciones o la modificación de las escaleras para poder cumplir los requisitos básicos de evacuación, promueven que el alumno vaya tomando poco a poco consciencia de la incidencia de todas las asignaturas técnicas a la hora de elaborar un proyecto y su estrecha relación con la expresión de la forma del proyecto.

Una de las ventajas de utilizar sistemas integrados de información y dibujo como es el caso de sistemas BIM, facilita y agiliza que las decisiones que se deban de tomar para la adecuación del proyecto a las nuevas necesidades sean adoptadas de forma única y de conjunto, evitando un de los errores habituales que se arrastran en los proyectos profesionales, es decir, la falta de coherencia entre los diferentes documentos que forman un proyecto.

Finalmente, el alumno deberá de dar repuesta a todos los requisitos planteados desde las diferentes áreas de conocimiento, obteniendo un proyecto que sea modificado lo menos posible de su planteamiento original, o al menos que las modificaciones sean coherentes con las intenciones iniciales. 


\section{Resultados}

Los resultados de aprendizaje obtenidos en esta metodología, mas allá de la documentación que se requiere a cada alumno de su proyecto para cumplir los requisitos técnicos que debe de cubrir su proyecto, es el inicio en un cambio en su mentalidad a la hora de enfrentarse aun nuevo proyecto. Ello es debido a que las modificaciones que en ocasiones debe de realizar en su propuesta inicial, conllevan cambios sustanciales en la propuesta inicial del proyecto, lo que requieren un esfuerzo importante en tiempo y recursos que no son considerados en la evaluación de la asignatura. Por tanto, el fin último de esta nueva propuesta metodológica, se basa en la necesidad de contemplar desde el momento inicial de la idealización del proyecto, tanto los requisitos funcionales, formales y técnicos, entendiendo la relación de todos ellos con el modo en que se percibe en la forma y el espacio del proyecto. (Herreros, 1997).

Las fases que atraviesan los estudiantes durante el proceso de aprendizaje responden fehacientemente a la curva del cambio basado en el modelo de Küber-Ross pero aplicadas a su proyecto, que pasa desde la negación y resistencia a las modificaciones que deben de hacer en su idealización inicial para que sea viable, debidas por ejemplo a la necesidad de introducir los medios de evacuación en caso de incendios o adaptar su estructura a la distribución de las plazas de aparcamiento de un garaje, hasta la exploración de nuevas posibilidades y la aceptación de dichos cambios por entenderlos necesarios e irrefutables. Debe entenderse el cambio no tanto como un parámetro de la metodología, sino como parte del propio aprendizaje.

Finalmente, a través del hilo conductor del proyecto arquitectónico los alumnos dan respuesta a la resolución técnica del proyecto, pero sin perder la identidad original del proyecto. Los estudiantes evidencian la intima relación entre las distintas áreas donde deben de convivir elementos tales como los huecos y patinillos de las instalaciones con la organización estructural de los forjados y su cálculo, o la instalación de saneamiento en concordancia con los planos constructivos de cimentación. 


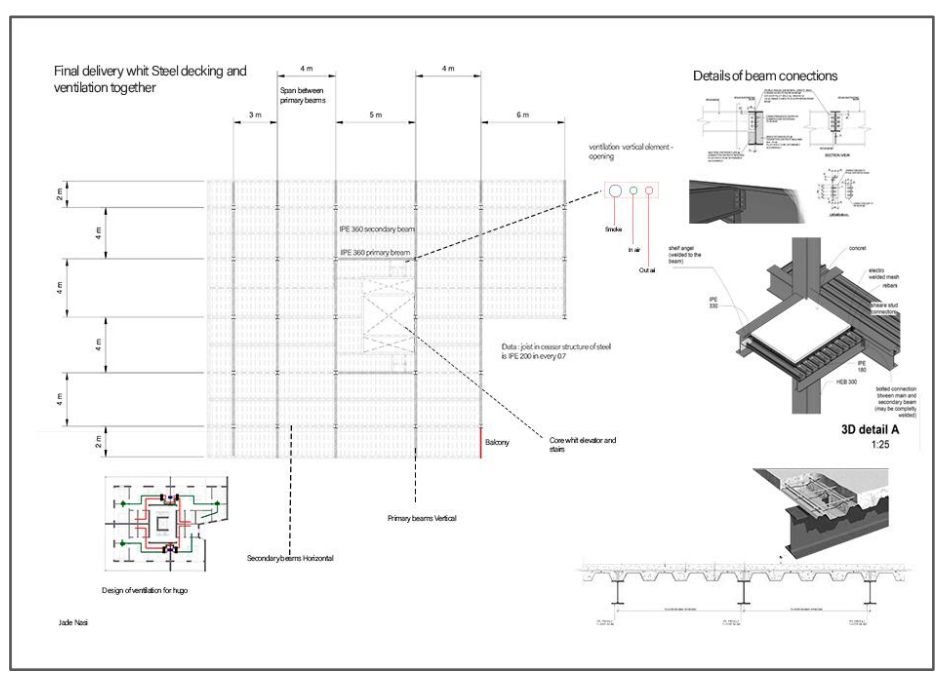

Fig. 2 Plano de alumno de $3^{\circ}$ curso donde se ha tenido en cuenta para su elaboración el cálculo estructura (estrucutras), la organización del forjado (construcción) y los huecos necesarios para instalaciones.

\section{Conclusiones}

Más allá de la obtención de una evaluación bajo rubricas que validan lo adecuado o no de los documentos obtenidos por los alumnos, el verdadero resultado del aprendizaje va más allá de una nota numérica. Se trata romper la visión sesgada que se sigue teniendo del proyecto arquitectónico y de concienciar al alumno de que en su idealización es necesario conocer la implicación de todas las áreas y su conexión a la hora de obtener un proyecto. (Ortega 2019)

Tras la aproximación al desarrollo técnico del proyecto arquitectónico, se ha preparado al alumno para que en el siguiente acercamiento en la idealización del proyecto, tenga presente incluso de forma involuntaria, todos aquellos aspectos técnicos que ha comprobado que tienen gran incidencia a la hora implantarlos en el proyecto. En otras palabras, la incidencia de la construcción de la técnica arquitectónica con un significado tanto material como formal. (Piñon, 2007)

Siguiendo el planteamiento vertical de esta metodología, el tercer año de implantación de esta metodología, los alumnos se encuentran preparados y son conscientes para enfrentarse al reto establecido; la integración de todas las asignaturas tanto tectónicas como proyectuales en la resolución de un proyecto arquitectónico, rompiendo la barrera física y mental de la compartimentación del aprendizaje por asignaturas, para adentrase en un 
nuevo escenario de aprendizaje multidisciplinario y colaborativo basado en proyectos, donde todas las áreas de conocimiento participan de forma coordinada en su resolución.

Pero el verdadero objetivo final es fomentar cambio de mentalidad a la hora de abordar el proyecto arquitectónico, donde el estudiante desarrolle las competencias que le permitan plantear y diseñar íntegramente todas las dimensiones del proyecto, desde su forma e implantación hasta su materialización técnica de forma solidaria, entendiendo la dimensión relevante que adquiere la construcción de la técnica en la génesis formal del proyecto.

Aunque no se ha realizado un sondeo específico sobre la irrupción de esta metodología entre el alumnado, tras tres años de su implantación y su puesta en funcionamiento en el último curso previo al enfrentamiento del alumno a su trabajo final de grado, alumnos y profesores han asimilado este nuevo planteamiento como lógico, comprobando los profesores el incremento del nivel técnico-proyectual de los proyectos así como el interés de las cuestiones planteadas por los alumnos en las correciones.

Volviendo a las palabras citadas iniciales de Ignacio Vicens, la intención última es que el alumno no entienda un proyecto como un pastel de capas inconexas e independientes, debe comprender que estas capas se encuentran estrechamente ligadas y forma parte de un elemento mayor: el proyecto arquitectónico.

\section{Referencias}

Anasagasti, T. (1995), La enseñanza de la arquitectura. Madrid, Ed Inst. Juan de Herrera.

Azua, F, (1988) El aprendizaje de la decepción. Ed. Pamiela.

e rreros e talles constructivos y otros fetiches perversos alencia diciones enerales de la o nstrucci $n$

Leon, I., Sagarna, M., Mora, F., Marieta, C., Otaduy, J. (2016) El empleo de la tecnología BIM en la docencia vinculada a la Arquitectura. Aprendizaje cooperativo y colaborativo basado en Proyecto reales entre diferentes asignaturas. IV Jornadas de Innovación Docente en Arquitectura. Pp 191197, Valencia

Lozano, J.M. (1994) La enseñanza de proyectos en España. Enseñar o aprender, en Arquitectura ${ }^{\circ}$ $297,31-34$, p. 34

Ortega, V. (2019). El detalle constructivo como expresión multisecular de la forma. VII Jornadas sobre Innovación Docente en Arquitectura, pp 702-714, Madrid.

Piñon, H. (2006). Teoría del proyecto. Barcelona: Edicions UPC.

Piñon, H. (2007). La arquitectura como materia de proyecto. Disponible en https://heliopinon.org/escritos_y_conferencias/det-la_arquitectura_como_materia_del_proyecto_i58185 